\title{
Factores de riesgo de malversación de fondos financieros en la Federación Peruana de Fútbol: 2015-2018 ${ }^{1}$
}

\author{
Hillary Milagros Torres Morales ${ }^{2}$ \\ Annie Stephanie Acosta Goñez $z^{3}$
}

Recibido: 23 de mayo de 2019

Aprobado: 4 de junio de 2019

\section{Clasificación JEL: D81, L83}

\section{Resumen}

El presente trabajo de investigación, expuesto en el "IV Congreso Latinoamericano de Estudiantes de Contaduría y Administración CONTAD 2019", está referido a la Federación Peruana de Fútbol (FPF) entre los años 2015 a 2018 en Lima, Perú, para dar a conocer el despilfarro de dinero que se cometió en este tiempo e identificar y numerar los hechos que sustentan las irregularidades causadas por la corrupción en esta entidad. Los objetivos son: determinar los factores de riesgo de malversación de fondos y sus principales efectos dentro de la Federación Peruana de Fútbol en el periodo 2015-2018, corroborar en qué medida la deficiente administración de los dirigentes de la Federación Peruana de

1 Torres Morales, H. M., \& Acosta Goñez, A. S. (2019). Factores de riesgo de malversación de fondos financieros en la Federación Peruana de Fútbol: 2015-2018. Revista Activos, 17(1), 15-49. DOI: https://doi.org/10.15332/25005278.5393

2 Estudiante de la Facultad de Ciencias Contables de la Universidad Nacional Mayor de San Marcos. Lima, Perú. Correo electrónico: hillary.torres1@unmsm.edu.pe.

3 Estudiante de la Facultad de Ciencias Contables de la Universidad Nacional Mayor de San Marcos. Lima, Perú. Correo electrónico: annie.22sag@gmail.com 
Fútbol afectó a la entidad y conocer los factores exógenos que perjudicaron la mencionada Federación. El diseño de la investigación es no experimental y de tipo transversal y descriptivo. Se hace uso de fuentes primarias como encuestas y entrevistas a representantes de la Controlaría General de la República del Perú y fuentes adicionales como es el Informe de Auditoría de Cumplimiento n. ${ }^{\circ}$ 03-2017-2-0217 "A los procesos de contrataciones" y Auditoría n. ${ }^{\circ}$ 08-2016-2-0217 "Al otorgamiento y utilización de recursos públicos a las federaciones deportivas nacionales", entre otros. Se concluye que la malversación de fondos afectó la buena marcha de la administración desde el punto de vista económico-financiero. Cabe destacar que debido a los últimos acontecimientos esta investigación es pionera en evidenciar los problemas de esta institución.

Palabras clave: corrupción, Federación Peruana de Fútbol, irregularidades financieras, riesgos.

\title{
Risk factors of embezzlement of financial funds in the Peruvian Football Federation: 2015-2018
}

\begin{abstract}
The present research, exposed at the IV Latin American Congress of Students of Accounting and Administration - CONTAD 2019, refers to the Peruvian Federation of Football (FPF) between 2015 to 2018 in Lima, Peru, for publicize the waste of money that was committed in these years and identify and number the facts that support the irregularities caused by corruption in this entity. The objectives are: to determine the risk factors of embezzlement and its main effects within the Peruvian Football Federation in the period 2015-2018, corroborate to what extent the poor administration of the leaders of the Peruvian Football Federation affected the entity and know the exogenous factors that affected the Peruvian Football Federation. The research design is non-experimental and cross-sectional and descriptive. It makes use of primary sources such as surveys and interviews with representatives of the Office of the Comptroller General of the Republic of
\end{abstract}


Peru and additional sources such as the Compliance Audit Report No. 032017-2-0217 “To the contracting processes", Audit No. 08-2016-2-0217 “To the granting and use of public resources to the national sports federations", among others. It concludes that the misappropriation of funds affected the good progress of the administration from an economic-financial point of view. It should be noted that due to the latest events this research is pioneer in evidencing the problems of this institution.

Keywords: Corruption, Peruvian Football Federation, Financial irregularities risks.

\section{Introducción}

La tecnología avanza a gran velocidad, lo que nos simplifica varias actividades. Tiene un rol importante en cada organización, que combinado con un sistema de control interno a medida podría garantizar un mínimo de riesgos. De aquí que la mayor vulnerabilidad de una organización es el elemento humano expuesto a diversos factores que lo llevarán a cometer fraude; se puede controlar la tecnología y las máquinas, pero controlar las motivaciones de una persona al momento de decidir entre lo positivo o negativo es imposible. Se encuentra la siguiente explicación:

La pirámide de Maslow es una teoría que considera las necesidades de una persona como combustible para su conducta. Se muestran cinco niveles ordenados por prioridad que en síntesis nos enseñan que a medida que vamos satisfaciendo necesidades básicas desarrollamos necesidades más elevadas. El motivo del fraude podría ubicarse en cualquier nivel impulsado por necesidades sociales, de seguridad, fisiológicas, de reconocimiento o autorrealización. (Reid-Cunningham, 2008, p. 51)

Según un caso de la Corte Suprema de Justicia en Arequipa encontramos como definición del fraude de malversación de fondos: "El cambio del destino de asignaciones de dinero y bienes que terminan no siendo usados para su misión inicial" (Recurso de Nulidad, 2005, p. 1). Aplicando la pirámide de Maslow, distintas necesidades motivan la malversación. 
Otro caso de relevancia internacional implicó al penúltimo presidente de la FPF, con más de diez años en el cargo. En 2015 fue investigado por asociación ilícita y varias irregularidades de la Federación Internacional de Fútbol Asociado (Fifa) por lo que fue retirado del cargo. Con el último presidente de la FPF también acontecerían una serie de irregularidades que empezarían en 2017, el año de la gestación de lo que en 2018 explotaría como uno de los mayores escándalos de corrupción en el país. Actualmente, ya es investigado por la Fiscalía Contra el Crimen Organizado.

El fútbol es la actividad que moviliza millones de personas sin distinciones de creencias, géneros, edades y niveles socioeconómicos. El deporte rey es regulado por la Fifa que a la fecha es la organización multilateral que mayor cantidad de países afiliados tiene, al ser global posee normas de obligatorio cumplimiento que permiten transparencia y equidad en las competencias. Estas normas se han ido perfeccionando con el tiempo; este es un proceso continuo que se nutre con los aportes de la ciencia y la tecnología pero que también cuenta con un componente humano.

Perú recién se encuentra en un proceso de formalización de la actividad, con un incipiente "derecho deportivo" así como una legislación no muy clara sobre faltas graves. Además de los hechos finales de corrupción vinculados a la Federación Peruana de Fútbol (FPF), se evidencia que sacar provecho de una organización con deficiente control interno puede haber sido estos últimos años la mina de oro de diversos funcionarios.

El tema es importante porque se demuestra los efectos de un deficiente control administrativo-financiero y sus consecuencias dentro de la Federación Peruana de Fútbol, visto desde la parte de control interno de la auditoría.

También es de interés nacional y con repercusión mundial porque la corrupción lamentablemente no es ajena en nuestro país y en la presente investigación hemos evidenciado lo arraigada que se encuentra en la Federación Peruana de Fútbol.

Y es viable porque existe suficiente información para llevar a buen término la investigación desarrollada. 


\section{Problema general}

¿Cuáles son los factores de riesgo de malversación de fondos financieros en la Federación Peruana de Fútbol: 2015-2018?

\section{Problemas específicos}

- ¿En qué medida la deficiente gestión administrativa, específicamente de los fondos financieros, afectó la FPF: 2015-2018?

- ¿Cuáles son los factores exógenos que perjudicaron el manejo de los fondos financieros en la Federación Peruana de Fútbol: 2015-2018?

\section{Objetivo general}

Determinar cuáles son los factores de riesgo de malversación de fondos financieros en la FPF: 2015-2018.

\section{Objetivos específicos}

- Analizar en qué medida la deficiente gestión administrativa de los fondos financieros afectó a la Federación Peruana de Fútbol: 2015-2018.

- Verificar cuáles son los factores exógenos que perjudicaron el manejo de los fondos financieros en la FPF: 2015-2018.

\section{Hipótesis general}

Los factores de riesgo de malversación de fondos afectaron la administración de la Federación Peruana de Fútbol en el periodo 2015-2018.

\section{Hipótesis específicas}

- La deficiente gestión administrativa de los fondos financieros perjudicó la dirección de la FPF: 2015-2018. 
- Los elementos exógenos de manejo de los fondos financieros afectaron la gestión de la Federación Peruana de Fútbol: 2015-2018.

Para una mejor comprensión de la investigación, el contenido se ha estructurado en cinco partes: Introducción, Metodología, Resultados, Conclusiones y Recomendaciones, y Referencias.

\section{Antecedentes}

El Mundial Rusia 2018 acabó y con su fin se despertaron varias dudas sobre los ingresos que se generaron en este periodo en los países participantes de este gran evento deportivo; esta incertidumbre tiene como respaldo las múltiples denuncias de corrupción que la Federación Internacional de Fútbol Asociado (Fifa) viene sumando. Toda esta información alentó la curiosidad de los medios periodísticos y la hinchada de cada país, pronto se abrieron investigaciones a las federaciones y los resultados fueron los esperados puesto que ya hace varios años es un secreto a voces el ocultamiento de delitos en nombre del fútbol.

Muchas de las investigaciones que existen se encuentran en proceso y otras ya dieron resultados; sin embargo, una característica en común de todas estas es la falta de información por parte de las federaciones, el hermetismo con el que se manejan y el apoyo de la Fifa para no esclarecer muchos casos.

En referencia a la búsqueda de información sobre el tema, se encontró en el diario La Nación lo siguiente: el presidente de la Confederación Brasileña de Fútbol (CBF), desde abril de 2015, Marco Polo Del Nero fue "suspendido de por vida de toda actividad ligada al fútbol" tanto nacional como extranjera, en septiembre de 2018, como consecuencia de la investigación que la Fifa le abrió en noviembre de 2015; entre sus cargos se encuentra el haber recibido sobornos de patrocinios deportivos por contratos de derechos televisivos (La Nación, 2018).

Por otra parte, en Colombia en el diario El Tiempo encontramos lo siguiente: el expresidente de la Federación Colombiana de Fútbol, Ramón 
Jesurún, salió expuesto en unos audios en los que participaba activamente en las negociaciones para asegurar el dinero de las entradas de los partidos eliminatorios del Mundial Rusia 2018. En este lío se vieron envueltos una boletería que vendería las entradas a mayor precio y la empresa alemana Adidas que le habría entregado dinero a la Federación para regresar como la marca patrocinadora de la selección durante el periodo 2013-2022; no se conoce el destino de todo el dinero (Deportes y Efe, 2017).

La Copa América es considerada por muchos la más grande fiesta del fútbol latinoamericano y para otros pocos su sueño y pesadilla. En 2015 la Fiscalía de Estados Unidos publicó una investigación que implicaba directamente a nueve autoridades de la Fifa y cinco empresarios de haber pactado sobornos por 150 millones de dólares a cambio de derechos de publicidad, patrocinio, transmisión y manipular sedes de campeonatos.

\section{Bases teóricas; explicación de casos y teorías}

\section{Federación Internacional de Fútbol Asociado (Fifa)}

Según los informes de la página web de la Fifa, entre 2015 y 2018 se muestra el incremento de ingresos gracias a sus diferentes estrategias; esta institución gobierna todas las federaciones de fútbol alrededor del mundo por lo que se encarga de organizar la Copa Mundial de Fútbol, los torneos olímpicos y otros campeonatos en sus múltiples categorías y variaciones (Federación Internacional de Fútbol Asociado - Fifa, 2018).

En la misma página también se encuentra que: todos estos eventos significan ingreso de dinero por derechos televisivos y radiales de transmisión, y derechos de patrocinios, entre otros, dejando grandes cantidades de dinero que son reinvertidas y repartidas a todas las federaciones del mundo; cuenta con 211 federaciones, posee 18 países afiliados y es la que mueve la mayor cantidad de dinero como deporte teniendo en cuenta que 3200 millones de telespectadores vieron en 2010 el Mundial Sudáfrica y el Mundial Brasil 2014, y los dígitos subieron en Rusia 2018 con 3572 millones de personas (Federación Internacional de Fútbol Asociado - Fifa, 2018). 


\section{Caso Manuel Burga}

El penúltimo presidente de la Federación Peruana de Fútbol, Manuel Burga, ha estado relacionado con actos de corrupción por cobrar millones de dólares por sobornos. Asumió su mandato el 4 de octubre de 2002, fue reelecto en 2007 y 2010, a pesar de que no era aceptado por la opinión pública. El testigo clave, Santiago Peña, quien fue empleado de la empresa de marketing deportivo Full Play, tuvo como prueba un archivo de Excel en el cual se detallaban pagos a ocho funcionarios de la Conmebol (Confederación Sudamericana de Fútbol). Se sabe que cada uno de ellos tenía un nombre clave que eran marcas de autos; de quien se habla usaba el apodo de Fiat y los pagos que recibía podían ser en efectivo o transferencias electrónicas. Otro testigo clave, cuya identidad no ha sido revelada por medidas de seguridad, menciona que Burga había pagado coimas de hasta 160 millones de dólares a los altos dirigentes de las federaciones. Y el tercer testigo que citamos es Alejandro Burzaco, empresario argentino quien declaró en el juicio del Fifagate, que se realizó en Estados Unidos.

\section{Instituto Peruano del Deporte (IPD)}

Siendo este el ente rector del deporte nacional, dependiente del Ministerio de Educación, no posee control sobre la Federación Peruana de Fútbol, por lo que no subvenciona al deporte y no tiene jurisdicción sobre cuestiones políticas y financieras de la selección. Sin embargo, posee autoridad para supervisar establecimientos, entrenamientos y demás; sucede que la FPF y el IPD son entes lejanos, así que aumentan los riesgos de fraude al no existir control.

\section{Federación Peruana de Fútbol (FPF)}

Según Altuve, uno de los primeros en investigar el fútbol como empresa, "el deporte como una empresa atípica aparece en 1982 con dos hechos importantes que son el crecimiento del patrocinio deportivo y la centralización de los derechos de comercialización de los Juegos Olímpicos" (Altuve, 2011, p. 51). 
Nos encontramos entonces ante una empresa peruana que mueve millones sin un adecuado control interno y no se puede dar una cifra exacta de cuánto dinero se mueve en la Federación; aun con la publicación de estados financieros no existe aval para su legitimidad puesto que las auditorías realizadas no arrojan opiniones contundentes. Las notas a los estados financieros no forman parte de las publicaciones y desde 2017 la Federación guarda hermetismo con su información, al solo publicar sus Estados de Resultados que en 2018 fueron inexistentes. En 2004 la FPF contrató los servicios de una firma de asesoría empresarial para que examinara la institución, y es así como Apoyo Consultoría titula el diagnóstico: "Desarrollo e implementación de una estrategia para la mejora de la competitividad e incremento de la rentabilidad en el fútbol". Los puntos resaltantes son la informalidad en las relaciones entre los entes de la trinidad deportiva (FPF-IPD-Fifa) y la falta de responsables y asignaciones para las diversas funciones básicas de la Federación.

\section{Caso Oviedo}

El último presidente de la FPF fue Edwin Oviedo quien es conocido por su reciente prisión preventiva debido a delitos relacionados con la corrupción. Asumió la presidencia de la FPF el 18 de diciembre de 2014 y lo más destacado de su mandato fue la clasificación de la selección peruana al Mundial Rusia 2018. Sin embargo, se vio opacado por su vínculo con la organización criminal "Los cuellos blancos del puerto", a la cual se le atribuye la autoría del asesinato de los dirigentes sindicales de la Cooperativa Tumán, una de las primeras azucareras en Lambayeque, Perú. Oviedo ocupaba cargos administrativos deficientes.

Entre los más destacados presuntos delitos, se encuentran:

- Tráfico de influencias: relacionado al exjuez supremo César Hinostroza, a quien le habría dado entradas al Mundial Rusia 2018, pasajes e incluso viáticos por un valor mayor a 8000 dólares.

- Cohecho activo específico: se sostiene que Oviedo se comprometió con César Hinostroza de manera extraordinaria a darle entradas 
para las eliminatorias al Mundial Rusia 2018 a cambio de favores judiciales. Como testigo se tiene al colaborador especial n. ${ }^{\circ} 409$ 2018 quien entregó dos CD de evidencia.

- Cohecho activo genérico: se maneja la información que Oviedo se habría comprometido con César Hinostroza en pagarle mensualmente 3300 soles para que lo asesore y sea desvinculado en el proceso judicial que tiene en Lambayeque, Chiclayo (Perú) con dinero de la FPF, por el caso de la Cooperativa Tumán (mencionado anteriormente).

- Y, por último, se deslinda de todo lo sucedido que Oviedo forma parte de la banda corrupta "Los cuellos blancos del puerto", quien los financiaba y daba importantes regalos.

Después del escándalo de corrupción que afectó al expresidente de la FPF, la Fiscalía de la Nación intervino la Videna de San Luis debido a poca facilidad de información por parte de la Federación. En síntesis, se logró encontrar en el informe, luego de la intervención, lo siguiente:

a. Reporte de ingresos y gastos del Mundial Rusia 2018: ingresos en dólares $\$ 9947500$ y en soles $S / 32667537.50$, señalando como total de gastos en dólares $\$ 14484434.06$ y en soles S/47 577713.71 y como déficit la suma de -\$4536 934.06 y -S/14 900176.21 cifras impresas en color rojo.

b. Reporte de ingresos y gastos por venta de entradas del Mundial Rusia 2018: ingresos en dólares $\$ 10314985.00$ y en soles $S / 33884725.73$, gastos en dólares $\$ 15124349.06$ y en soles S/49 679 834.49, y como déficit la suma de $-\$ 44809364.06$ y $-S / 15795108.76$ cifras impresas en rojo.

c. Ingresos de la Fifa: en dólares \$9307 500 y en soles S/30 575137.50 .

En cuanto a los demás informes de ingresos y gastos, todas estas hojas no contaban con sellos ni formas, sin embargo, tenían el logo de la 
Federación; se encontraron también varias tarjetas de presentación de ministros, exministros, abogados, contadores, etc. Muchos de ellos implicados en corrupción. Con un enfoque basado en riesgos, se analiza la Federación de arriba abajo y, especialmente, sus procesos; en estos está el riesgo y en este caso la malversación. 


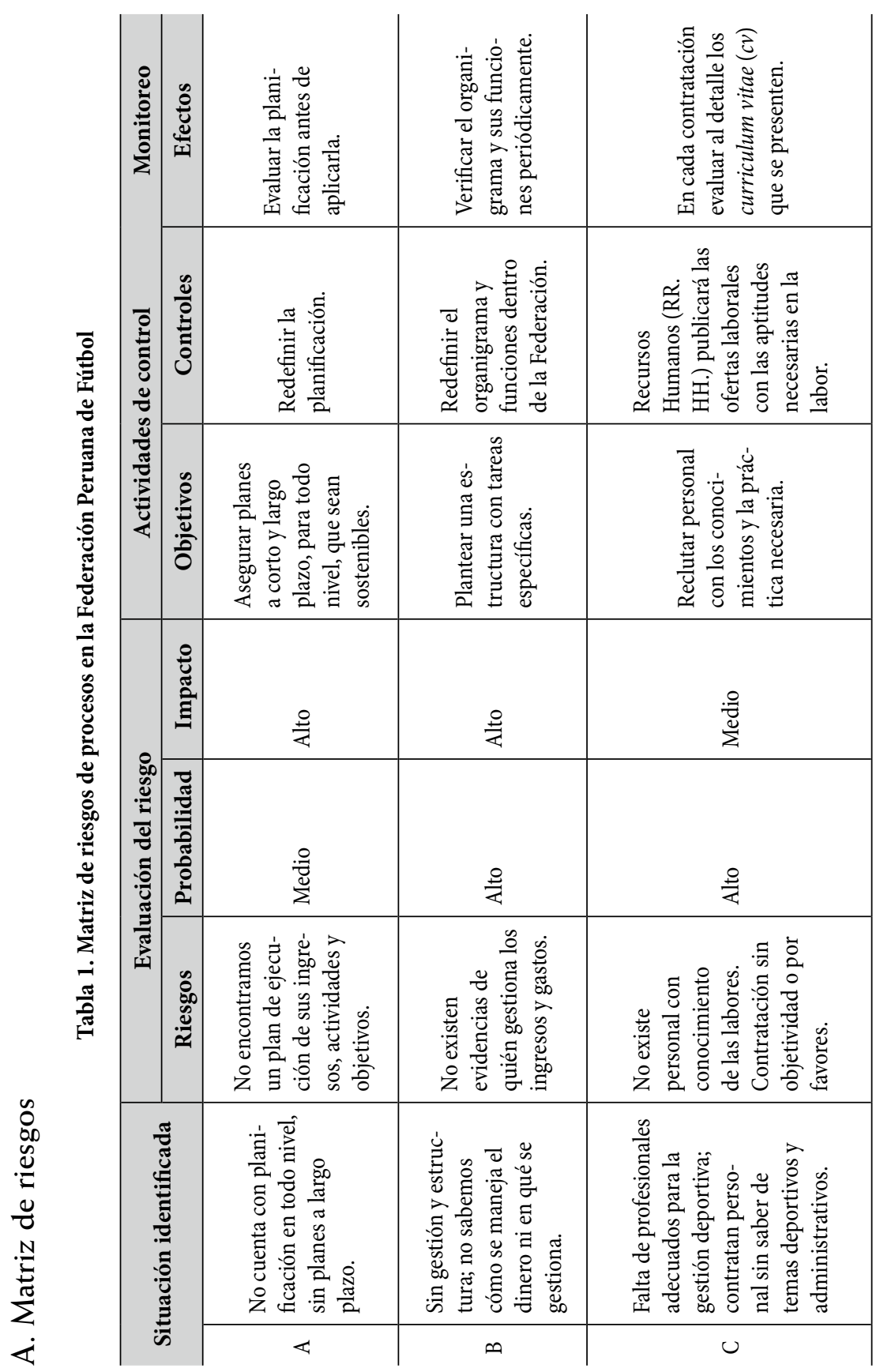

Universidad Santo Tomás, Facultad de Contaduría Pública 
Factores de riesgo de malversación de fondos financieros $\mid$ REVISTA ACTIVOS 27 en la Federación Peruana de Fútbol: 2015-2018

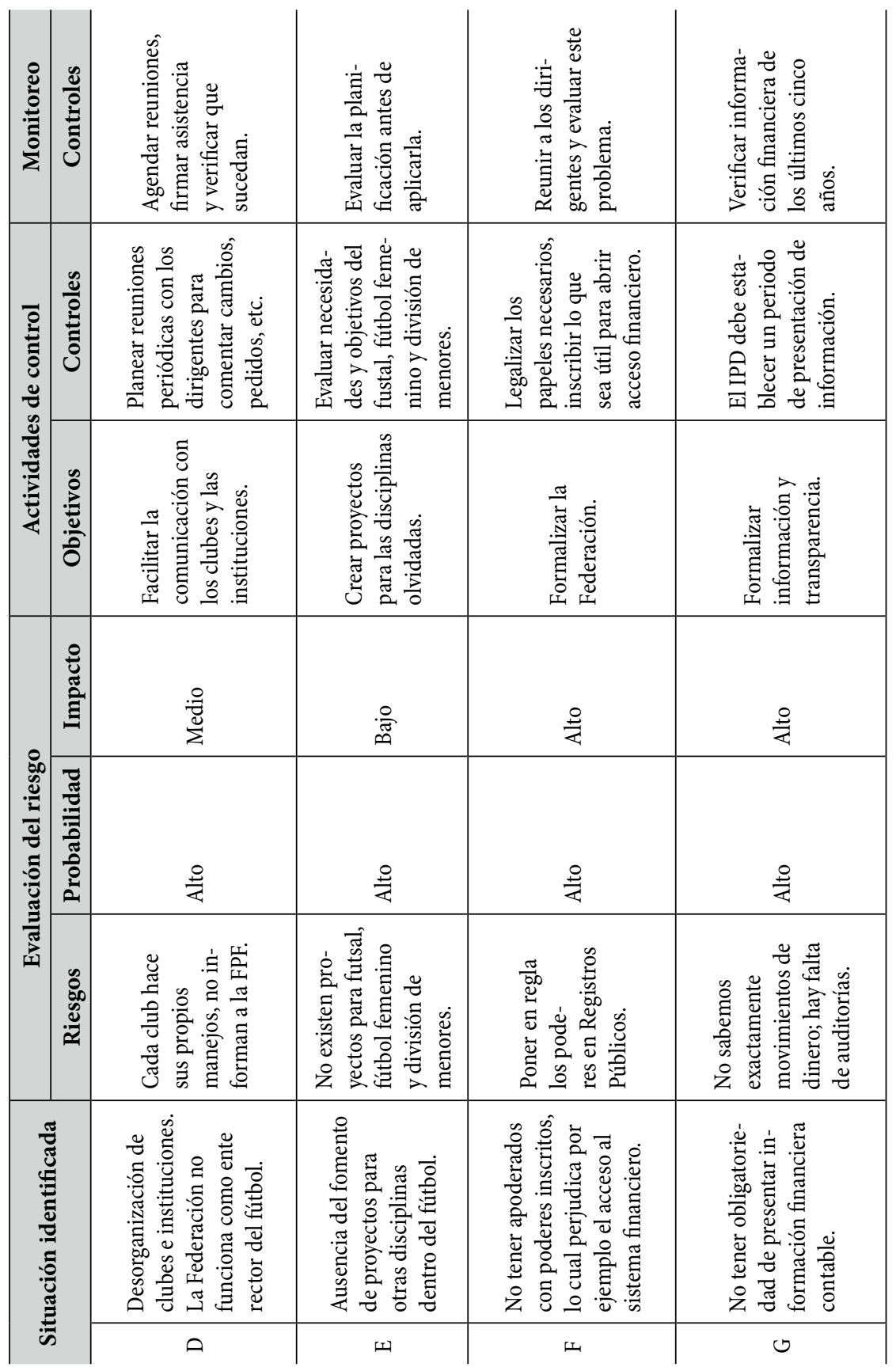




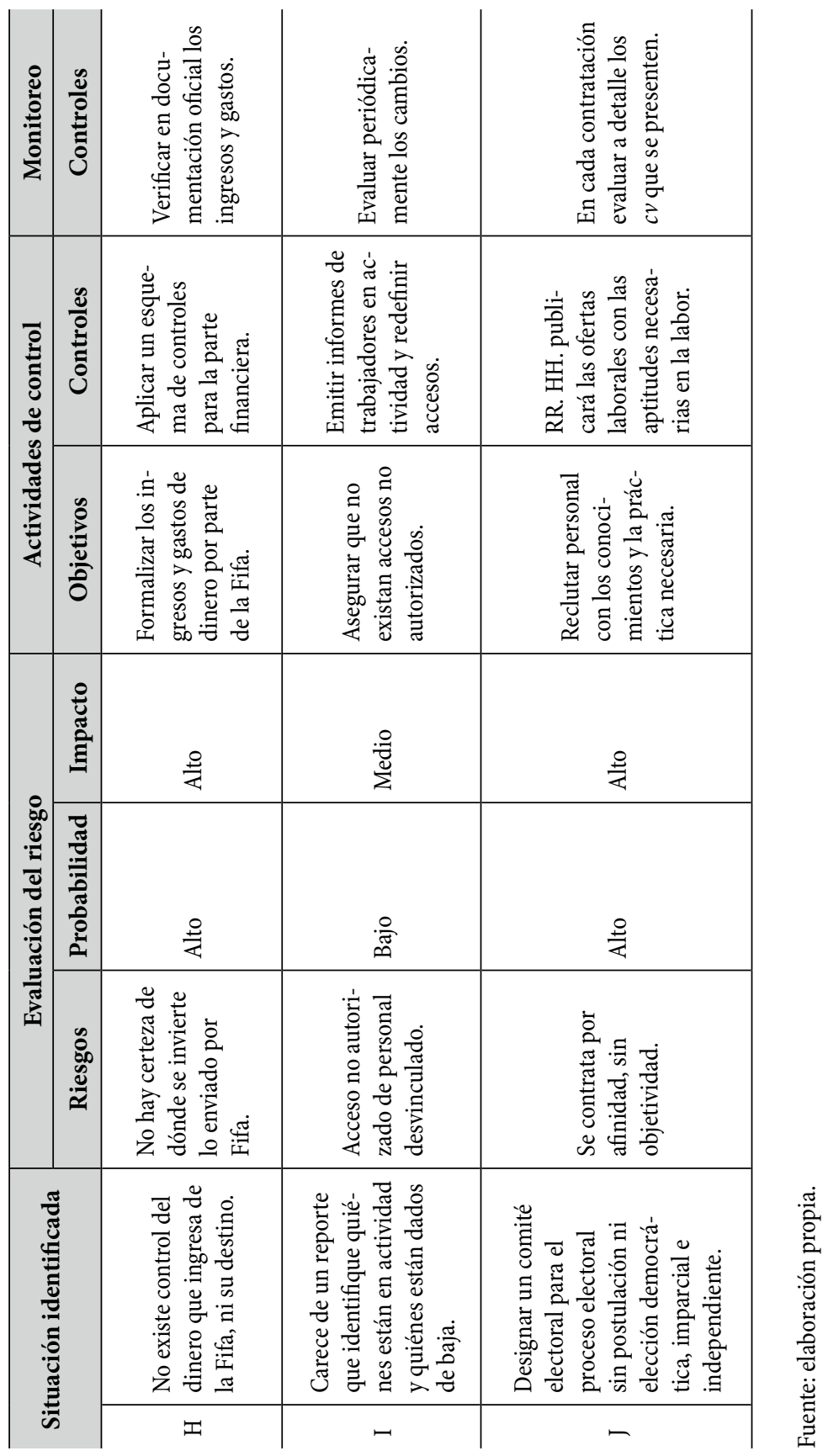

Universidad Santo Tomás, Facultad de Contaduría Pública 


\section{B. Efectos - matriz de riesgos}

Los efectos de la malversación de fondos en la FPF, dada la matriz de riesgos y los casos de corrupción, dan como resultado la pobre mirada extranjera al fútbol nacional. Este riesgo permite que el dinero no se use en los objetivos que tiene la Federación la cual, como institución del deporte, no invierte de una correcta manera y el dinero no llega a todas las ramificaciones del deporte. Los factores externos perjudiciales provienen de la falta de control de salida de dinero por parte de la Fifa.

\section{Marco conceptual}

Control interno: según el Ministerio de Economía y Finanzas (MEF), así como la Contraloría General de la República del Perú: "Es el conjunto de actividades, planes, normas, procedimientos, métodos y demás, cuya estructura la incluyen actitudes de autoridades y personal organizados con el objetivo de prevenir posibles riesgos" (MEF, 2019).

Corrupción: para el portal web Proética Anticorrupción es: "Cuando una persona actúa sin orientarse por la confianza que se ha depositado en su desempeño, a través de la posición o cargo determinado, y consigue beneficios para ella o para terceros que, de otra manera, no se habrían generado" (Proética, 2011).

Desviación de auditoría: se refiere a que

Se emiten las desviaciones de Cumplimiento para determinar la responsabilidad funcional, Civil y Penal y se mencionan las deficiencias y las debilidades en el control interno detectadas por el auditor. Por lo tanto, abarca los hechos y otras informaciones obtenidas que merecen ser comunicados a los funcionarios de la entidad auditada y a otras personas interesadas. (Contraloría General de la República, 2017) 
Malversación: de acuerdo con la Corte Suprema de Justicia en el caso RR n. ${ }^{\circ} 100-04:$ "Nos describe el comportamiento típico de este delito como el cambio del destino de asignaciones de dinero y bienes que terminan no siendo usados para su misión inicial" (Recurso de Nulidad, 2005).

\section{Metodología}

\section{Tipo y diseño de investigación}

La presente investigación es de tipo no experimental ya que no se ha dado situación alguna que influya a manera de tratamiento en los hechos estudiados; y de corte transversal porque se determina a las observaciones, independientes del tiempo. Tiene un enfoque de tipo cuantitativo y descriptivo, porque se busca conocer el impacto de los riesgos de malversación de fondos y sus efectos administrativos en la Federación Peruana de Fútbol.

\section{Unidad de análisis}

Datos de la encuesta elaborada por los autores, que consta de once preguntas específicas al tema de riesgo de malversación de fondos y sus efectos en la gestión administrativa de la Federación Peruana de Fútbol 2015-2018.

\section{Población de estudio}

Está constituida por la FPF.

\section{Muestra}

La muestra se determinó en función del alcance y las restricciones de la investigación, en este caso la Federación Peruana de Fútbol. 


\section{Recolección de datos y procesamiento}

La información procesada se tomó de los estados financieros de la FPF que aparecen en la página web de la Superintendencia de Banca, Seguros y AFP (SBS). Cabe mencionar que solo es pública la de los años 2015, 2016 y 2017. También se toman como referencia, informes de auditorías de la Contraloría General de la República de los años mencionados. Así mismo, se procesa la información de la encuesta elaborada que arroja datos estadísticos importantes, gracias al programa SPSS (se anexa al trabajo).

- Técnica de recolección de datos: encuesta.

- Población: 30 personas elegidas por su conocimiento en la materia.

- Muestra: 20 personas.

- Método de análisis: estadística descriptiva.

\section{Criterios de inclusión}

- Que pertenezcan a la Universidad Nacional Mayor de San Marcos (UNMSM).

- Que los encuestados pertenezcan a la Facultad de Ciencias Contables.

- Que hablen español.

- Que tengan conocimiento actualizado sobre el tema tratado.

\section{Criterios de exclusión}

- Que no pertenezcan a la Facultad de Ciencias Contables.

- Que presenten algún tipo de trastorno psiquiátrico o diversidad cognitiva.

- Quienes no deseen participar. 


\section{Resultados}

Según los informes de la página web de la Fifa, entre 2015 y 2018 (tabla 1) se muestra el incremento de ingresos gracias a sus diferentes estrategias; esta institución gobierna todas las federaciones de fútbol alrededor del mundo por lo que se encarga de organizar la Copa Mundial de Fútbol, los torneos olímpicos y otros campeonatos en sus múltiples categorías y variaciones (Federación Internacional de Fútbol Asociado - Fifa, 2018).

Estos son los reportes anuales de ingresos de la Fifa:

Tabla 2. Desglose de ingresos en millones de dólares

\begin{tabular}{l|c|c|c|c|c}
\hline Ingresos & $\mathbf{2 0 1 5}$ & $\mathbf{2 0 1 6}$ & $\mathbf{2 0 1 7}$ & $\mathbf{2 0 1 8}$ & Total \\
\hline Derechos de televisión & 258 & 96 & 209 & 2437 & 3000 \\
\hline Derechos de marketing & 157 & 115 & 261 & 917 & 1450 \\
\hline $\begin{array}{l}\text { Derechos de explotación de } \\
\text { licencias }\end{array}$ & 54 & 2014 & 48 & 60 & 2176 \\
\hline $\begin{array}{l}\text { Derechos de ventas de } \\
\text { entradas }\end{array}$ & 0 & 0 & 45 & 530 & 575 \\
\hline Otros & 78 & 87 & 51 & 52 & 268 \\
\hline Total & 547 & 2312 & 614 & 3996 & \\
\hline
\end{tabular}

Fuente: elaboración propia con base en informe de la Fifa.

Los ingresos que la Fifa recibe por diferentes tipos de conceptos se muestran también en la figura 1, siendo los derechos televisivos el rubro con mayor participación en los ingresos que generan. La diferencia entre los años 2015 y 2018 es abismal puesto que este último fue un punto clave ya que se celebró el Mundial de Rusia. La Fifa establece montos para cada país participante y estas sumas crecen con cada mundial; así, para Rusia 2018 se destinaron $\$ 791$ millones de dólares para premios y gastos de la organización, y un millón y medio más para la federación que alcanzara la fase final. Por otro lado, en Rusia 2018 la Fifa le pagó a cada club que cedió 
a un jugador a la Copa del Mundo, 8530 dólares por cada día que participó en el torneo. Este periodo de pago por día correspondió desde dos semanas antes del encuentro inaugural hasta el día después de que la selección para la cual juega el futbolista quedara eliminada de la competencia. Todo este dinero fue movido sin supervisión.

Figura 1. Desglose de ingresos de la Fifa: 2015-2018

DERECHOS DE
TELEVISIÓN
DERECHOS DE MARKETING
DERECHOS DE
EXPLOTACIÓN DE
LICENCIAS
DERECHOS DE VENTAS DE
ENTRADAS
OTROS
TOTAL

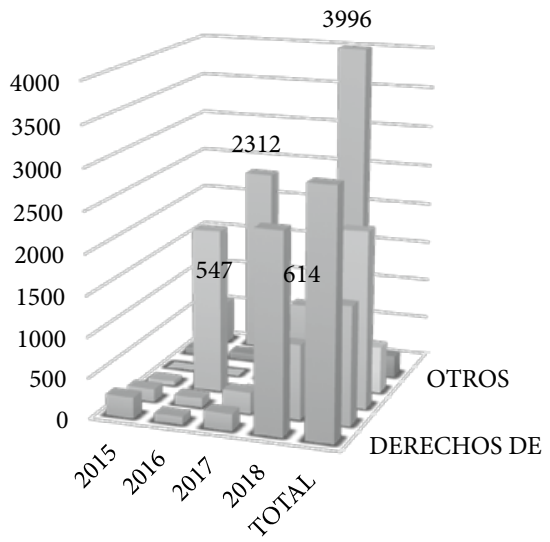

Fuente: elaboración propia con base en informe de la Fifa.

Tabla 3. Estado de resultados de la FPF 2015-2018

\begin{tabular}{|c|c|c|c|c|}
\hline Ingresos & 2015 & 2016 & 2017 & 2018 \\
\hline \multicolumn{5}{|c|}{ Ingresos ordinarios } \\
\hline Federación & S/14 773355.00 & S/17 725487.00 & S/17 436791.00 & - \\
\hline Mayores & S/39 952482.00 & $\mathrm{~S} / 28175074.00$ & $\mathrm{~S} / 42459146.00$ & - \\
\hline Menores & \multirow{3}{*}{ S/3 040865.00} & S/1 401444.00 & S/1 036618.00 & - \\
\hline Futsal & & $S / 175000.00$ & S/273 590.00 & - \\
\hline Otros servicios & & S/516967.00 & S/676246.00 & - \\
\hline
\end{tabular}




\begin{tabular}{|c|c|c|c|c|}
\hline Ingresos & 2015 & 2016 & 2017 & 2018 \\
\hline $\begin{array}{l}\text { Total ingresos } \\
\text { ordinarios }\end{array}$ & $S / 57766702.00$ & S/47993972.00 & S/61 882391.00 & - \\
\hline \multicolumn{5}{|c|}{ Otros ingresos de gestión } \\
\hline Ingresos por ley & S/6 980323.00 & S/1 276648.00 & S/6 304470.00 & - \\
\hline Aportes CSF y Fifa & $\mathrm{S} / 4772074.00$ & S/4 472293.00 & S/1 453060.00 & - \\
\hline Otros ingresos & S/1 412686.00 & S/1 075652.00 & S/1 480042.00 & - \\
\hline $\begin{array}{l}\text { Ingresos } \\
\text { financieros }\end{array}$ & S/5 163388.00 & S/2 305955.00 & S/1 007111.00 & - \\
\hline $\begin{array}{l}\text { Total otros } \\
\text { ingresos de gestión }\end{array}$ & $\mathrm{S} / 18328471.00$ & S/9 130548.00 & $S / 10244683.00$ & - \\
\hline $\begin{array}{l}\text { Total ingresos } \\
\text { del periodo }\end{array}$ & S/76 095173.00 & S/57 124520.00 & S/72 127074.00 & - \\
\hline \multicolumn{5}{|l|}{ Gastos } \\
\hline Menores & S/3 885505.00 & S/4 781525.00 & $\mathrm{~S} / 4716938.00$ & - \\
\hline Femenino & - & S/1 086335.00 & $S / 1071661.00$ & - \\
\hline Futsal & S/788 269.00 & S/1 070507.00 & S/1 056047.00 & - \\
\hline $\begin{array}{l}\text { Campeonato } \\
\text { reservas }\end{array}$ & S/3 395410.00 & S/6 800181.00 & S/6 436806.00 & - \\
\hline Mayores & S/29 233261.00 & S/18 975131.00 & S/27 176114.00 & - \\
\hline Federación & S/16 549298.00 & S/12 921217.00 & S/18906 665.00 & - \\
\hline Provisiones & $\mathrm{S} / 5415307.00$ & S/1 190892.00 & $S / 2783134.00$ & - \\
\hline Total gastos & S/59 267050.00 & $\mathrm{~S} / 46825788.00$ & S/62 147365.00 & - \\
\hline $\begin{array}{l}\text { Resultado del } \\
\text { ejercicio }\end{array}$ & $S / 16828123.00$ & $S / 10298732.00$ & S/9 979709.00 & - \\
\hline
\end{tabular}

Fuente: elaboración propia con base en informe de la FPF.

Según lo observado en la tabla 3, se aprecia que el resultado del ejercicio por año viene disminuyendo, debido a un problema entre la Superintendencia Nacional de Aduanas y de Administración Tributaria y el club de fútbol profesional Universitario de Deportes. Cabe precisar que 
en 2018 la FPF recibió la suma de S/11 814938.00 por parte de la Fifa, cifra que no se evidencia en los estados financieros y se desconoce la utilización de dichos fondos.

Por las cifras que se aprecian en los estados financieros, se puede argumentar que en la Fifa no existe un adecuado manejo de los fondos, hecho que se sustenta en varios factores, tales como:

- La falta de un buen registro contable.

- La deficiente gestión de los gastos que no son optimizados.

- La carencia de nota en los estados financieros, por lo que no se fundamenta la asignación de gastos o la sustentación particularizada de los ingresos, lo cual genera un descalce financiero.

- Los movimientos de ingresos y gastos que cuentan con comprobantes no son debidamente auditados.

- La carencia de control interno, lo cual ocasiona que no se ejecuten acciones de control a sus procesos financieros y administrativos.

Todos estos factores que al final de cuentas se traducen en un deficiente manejo de los fondos y así mismo un descontrol administrativo, impulsa los riesgos que son propios de una organización que incurre en limitadas acciones de control interno.

Complementariamente a las cifras presentadas, y también para dar mayor robustez al trabajo de investigación, se muestra el resultado de una encuesta formulada a docentes universitarios especialistas en auditoría y estudiantes de los últimos años de esta misma carrera, con conocimiento sobre las acciones administrativas de la FPF. Se empleó la escala de Likert para la estructuración de la encuesta y resultaron las categorías:

$1=$ Totalmente en desacuerdo $2=$ En desacuerdo $3=$ Ni de acuerdo, ni en desacuerdo; $4=$ De acuerdo y $5=$ Totalmente de acuerdo. 


\section{Resultados por preguntas}

Tomando en cuenta la encuesta realizada con una población de treinta personas y una muestra de veinte, entre alumnos y profesores conocedores del tema, obtuvimos una data transparente con información fidedigna, por lo cual pudimos desarrollar los siguientes gráficos para mayor entendimiento de nuestro asunto; cabe mencionar que los gráficos están basados en las once preguntas hechas en la encuesta, que están debidamente clasificadas en las cuatro dimensiones pertenecientes a nuestras variables.

Las siguientes preguntas pertenecen a la dimensión "Deficiente uso de los fondos de la FPF" que hacen parte de la variable independiente:

MF2: pregunta 10

¿Piensa usted que se hace un correcto y transparente manejo de los fondos que obtiene la FPF?

MF1: pregunta 11

En su opinión, ¿¿cree usted que existen tipos de fraude que pueden generar malversación de fondos en la FPF?

Figura 2. Dimensión Deficiente uso de los fondos en la FPF

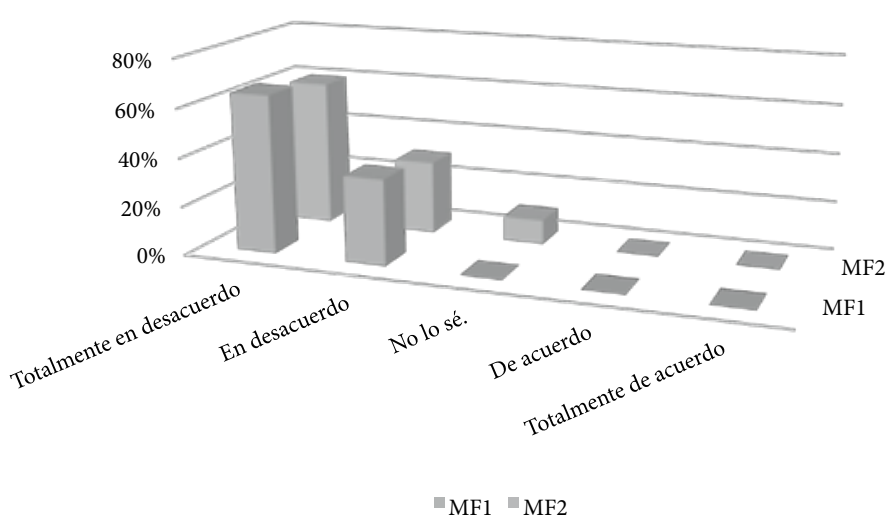

Fuente: elaboración propia (SPSS) 


\section{Interpretación:}

Las preguntas 10 y 11 (adjuntadas en los anexos), se refieren a la dimensión Malversación de fondos. En esta se halla que los encuestados en su mayoría están totalmente en desacuerdo con el desempeño financiero en la FPF, con un porcentaje que oscila entre $60 \%$ y $65 \%$.

\section{CF1: pregunta 7}

$¿$ Cree usted que en la FPF se tengan adecuadas calificaciones, conocimientos, habilidades y entrenamientos necesarios para realizar su trabajo adecuadamente?

CF2: pregunta 8

En su opinión, ¿ise realiza un adecuado control en los procedimientos administrativos en la FPF?

CF3: pregunta 9

¿Considera usted que se respeta la jerarquía en la FPF?

\section{Figura 3. Dimensión Escaso control en la FPF}

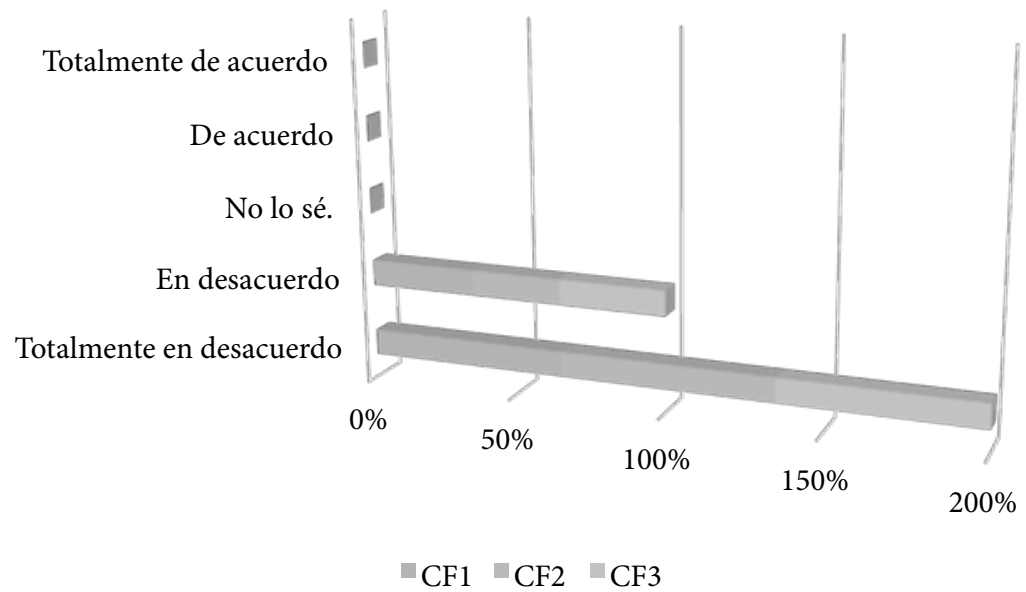

Fuente: elaboración propia (SPSS). 


\section{Interpretación:}

Las preguntas 7, 8 y 9 (adjuntadas en los anexos) se refieren a la dimensión Escaso control de fondos en la FPF. Los encuestados en su mayoría están totalmente en desacuerdo con el control que se tiene en la Federación Peruana de Fútbol, con un porcentaje que oscila entre el $65 \%$ y $70 \%$. La pregunta 8 tiene el mayor porcentaje de quienes están totalmente en desacuerdo.

GF1: pregunta 5

¿La importancia/impacto y similitud/probabilidad del riesgo de fraude financiero que ocurre en la FPF lo considera como parte de la corrupción de nuestro país?

GF 2: pregunta 6

¿Las acciones del presidente de la FPF son consistentes con los valores declarados y la conducta de la organización, como se espera de todos los demás empleados?

Figura 4. Dimensión Efectos en la gestión financiera en la FPF

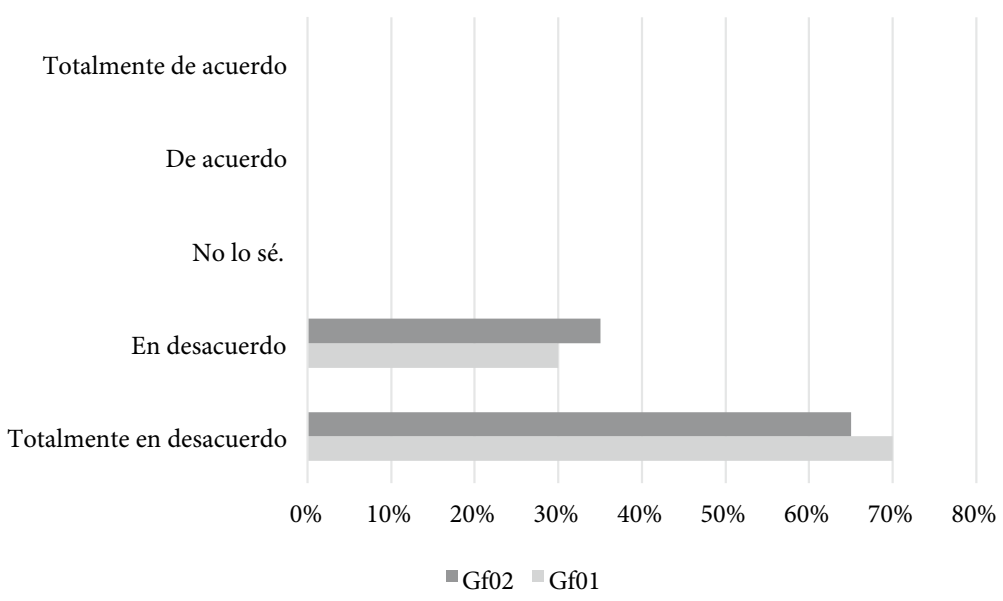

Fuente: elaboración propia (SPSS). 
Interpretación:

Las preguntas 5 y 6 (adjuntadas en los anexos) se refieren a la dimensión Efectos en la gestión financiera en la FPF. Los encuestados en su mayoría están totalmente en desacuerdo, con un porcentaje de entre $65 \%$ y $70 \%$. La pregunta 5 tiene el mayor porcentaje de quienes están totalmente en desacuerdo.

RR. HH. 1: pregunta 1

¿La alta dirección de la FPF demuestra altos estándares éticos?

RR. HH. 2: pregunta 2

¿La alta dirección de la FPF cumple con las leyes, las normas y los reglamentos que afectan a la organización?

RR. HH. 3: pregunta 3

$¿$ Cree usted que los directivos y empleados son sensibles a las consideraciones éticas y al impacto y la percepción de los demás a la hora de tomar decisiones o emprender acciones?

RR. HH. 4: pregunta 4

¿Piensa usted que la oficina y los jefes ponen el énfasis apropiado en la importancia de la integridad, la conducta ética, la justicia y la honestidad en sus relaciones con los empleados? 
Figura 5. Dimensión Efectos en la gestión administrativa en la FPF

Totalmente de acuerdo

De acuerdo

No lo sé.

En desacuerdo

Totalmente en desacuerdo

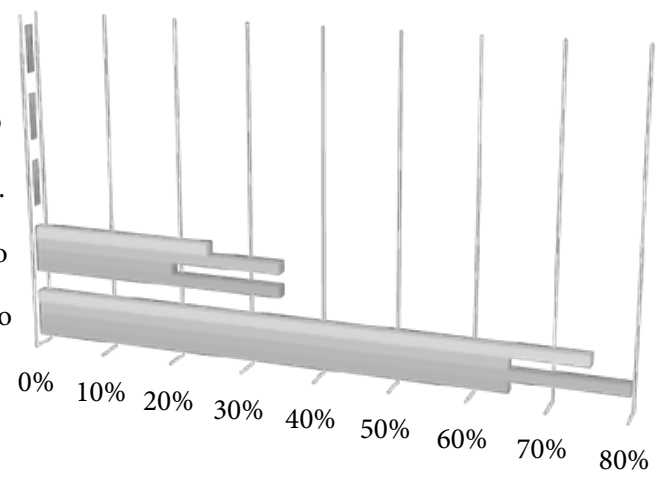

Rrhh04 Rrhh03 Rrhh02 Rrhh01

Fuente: elaboración propia (SPSS).

Interpretación:

Las preguntas 1, 2, 3 y 4 (adjuntadas en los anexos) se refieren a los Efectos en la gestión administrativa en la FPF, enfocados al rubro de recursos humanos (RR. HH.) en la Federación Peruana de Fútbol. Observamos que los encuestados en su mayoría están totalmente en desacuerdo, con un porcentaje que está entre el $65 \%$ y el $80 \%$. La pregunta 2 es la que tiene el mayor porcentaje de quienes están totalmente en desacuerdo.

Para efectos de validar la hipótesis se realiza la siguiente prueba estadística:

\section{Comprobando la hipótesis}

Correlación entre riesgo de malversación de fondos y sus efectos en la gestión administrativa en la FPF. Para el análisis de reciprocidad entre las variables mostradas en la tabla 3 , se propuso la siguiente hipótesis: 
H0: los factores de riesgo de malversación de fondos no afectaron la gestión de la Federación Peruana de Fútbol en el periodo 2015-2018.

Tabla 4. Correlación entre las variables

\begin{tabular}{l|l|l|c|c}
\cline { 3 - 4 } & \multirow{2}{l}{} & KMalFon & KGeAdm \\
\hline \multirow{3}{*}{$\begin{array}{l}\text { Rho de } \\
\text { Spearman }\end{array}$} & KMalFon & Coeficiente de correlación & 1.000 & $.820^{* *}$ \\
\cline { 3 - 5 } & & Sig. (bilateral) &. & .000 \\
\cline { 3 - 5 } & \multirow{2}{*}{ KGeAdm } & Coeficiente de correlación & $.820^{* *}$ & 1.000 \\
\cline { 3 - 5 } & Sig. (bilateral) & .000 &. \\
\cline { 3 - 5 } & $\mathrm{N}$ & 20 & 20 \\
\hline
\end{tabular}

**. La correlación es significativa en el nivel 0,01 (bilateral).

Fuente: elaboración propia (SPSS).

En la tabla 4 se observa que, en opinión de los encuestados, el valor de Sig. (bilateral) es 0.00 , lo cual evidencia que se cumple la $\mathrm{H} 1$; es decir, existe una fuerte correlación (82 \%) entre las variables Riesgo de malversación de fondos y Efectos en la gestión administrativa.

\section{Conclusiones y recomendaciones}

\section{Conclusiones}

En los factores de riesgo encontrados tenemos el deficiente uso de los fondos y el escaso control de ellos. El primero mencionado hace referencia a una mala administración en el dinero recaudado por la Federación Peruana de Fútbol, como vemos en las cifras de ingresos de los años 2015 a 2018, lo cual no refleja una adecuada y sostenida administración debido no solo a las autoridades corruptas que laboran en ella, sino también a una falta de control en la administración. En este sentido, no se cumple con la jerarquía en esta institución y los procedimientos que deberían seguirse son omitidos. Sus efectos ya han sido comprobados con base en una hipótesis, y mediante 
la encuesta realizada podemos decir que los riesgos de malversación de fondos sí desencadenan efectos negativos que repercuten en la gestión financiera y económica de la FPF.

Con los casos ya mencionados de corrupción de los últimos presidentes de la Federación Peruana de Fútbol, se pone en evidencia la falta de transparencia y compromiso con la administración de los dirigentes, ambos procesados por la justicia peruana. Así mismo, la gestión financiera y económica no refleja resultados aceptables ni fidedignos ya que sus estados financieros auditados no poseen opiniones contundentes ni existen notas en tales estados, lo cual no se puede considerar como un aval de la gestión administrativa. Además de ello, hay falta de responsabilidad con el dinero que ingresa cada año por medio de la Fifa y poca información pública, ya que no existen estados financieros desde 2017.

En los factores exógenos encontramos que el Instituto Peruano del Deporte, como ente de autoridad que evalúa correctamente el desempeño de todas las instituciones ligadas al deporte, es ajena a la supervisión de la FPF, lo cual dificulta un buen control de ella debido a que no se someten a una auditoría como las demás instituciones. Esto reflejaría la disfunción entre ingresos y gastos, la omisión del control interno de procedimientos y la corrupción de las autoridades que caen en las ya mencionadas "coimas" como parte de una vulnerada administración.

\section{Recomendaciones}

Se busca mediante un filtro que exista una elección democrática y correcta para la elección de sus autoridades, con la finalidad de que sean autoridades competentes y no se repitan los errores pasados con el tema de corrupción. También se sugiere que los procedimientos administrativos deben estar pautados en un manual para saber cómo actuar ante problemas similares a los anteriores y qué se debe hacer frente a ellos. Se recomienda que los estados financieros sean auditados por una entidad responsable, transparente y con prestigio, sin faltas de notas en ellos, que sus opiniones sean contundentes y 
que sean accesibles para el público. Estos deben ser publicados en la página web de la Superintendencia de Banca, Seguros y AFP (SBS) y debe haber un registro de cada ingreso y egreso realizado por la Federación Peruana de Fútbol, el cual deberá estar firmado por el jefe de cada área en la organización. Se aconseja que se formalice la relación con el Instituto Peruano del Deporte como órgano de control y supervisión, así como lo es para las demás instituciones del rubro del deporte, en busca de un mejor control interno en la FPF para que existan reportes de ello.

\section{Referencias}

Altuve, E. (2011). Consolidación del modelo deportivo globalizador transnacional. Espacio Abierto, 20(3), julio-septiembre, 501-517. Maracaibo, Venezuela: Universidad del Zulia. Recuperado de http://www.redalyc.org/ pdf/122/12219464007.pdf

Contraloría General de la República. (11 de julio de 2017). Capítulo VI. Ejecución del trabajo. Recuperado de http://www.contraloria.gob.ec/documentos/normatividad/MGAG-Cap-VI.pdf

Deportes y Efe. (21 de julio de 2017). Colfútbol y su presidente son mencionados en escándalo de corrupción. El Tiempo. Recuperado de https://www.eltiempo. $\mathrm{com} /$ deportes/futbol-internacional/federacion-colombiana-es-mencionadoen-escandalo-de-corrupcion-en-espana-111212

Federación Internacional de Fútbol Asociado - Fifa. (10 de enero de 2018). La Fifa. Recuperado de https://es.fifa.com/about-fifa/home-of-fifa/index.html

La Nación. (27 de abril de 2018). La Fifa suspendió de por vida al expresidente de la Confederación Brasileña de Fútbol por corrupción. La Nación. Recuperado de https://www.lanacion.com.ar/2129604-la-fifa-suspendio-de-por-vida-alex-presidente-de-la-confederacion-brasilena-de-futbol-por-corrupcion

MEF. (10 de febrero de 2019). Sistema de Control Interno. Ministerio de Economía y Finanzas del Perú. Recuperado de https://www.mef.gob.pe/es/ sistema-control-interno

Proética. (2011). Red Nacional Anticorrupción. Recuperado de http://www.redanticorrupcion.pe/ 
Recurso de Nulidad. (19 de enero de 2005). $R N n .{ }^{\circ} 100-04$ Arequipa. Corte Suprema de Justicia. Segunda Sala Penal Transitoria. Recuperado de http://idehpucp. pucp.edu.pe/images/documentos/anticorrupcion/jurisprudencia/recurso_nulidad_n100-2004_19-01-2005.pdf

Reid-Cunningham, A. (3 de diciembre de 2008). Maslow's theory of motivation and hierarchy of human needs: a critical analysis. Recuperado de https:// es.scribd.com/document/44303357/8703989-Maslows-Hierarchy-of-Needsa-Critical-Analysis 


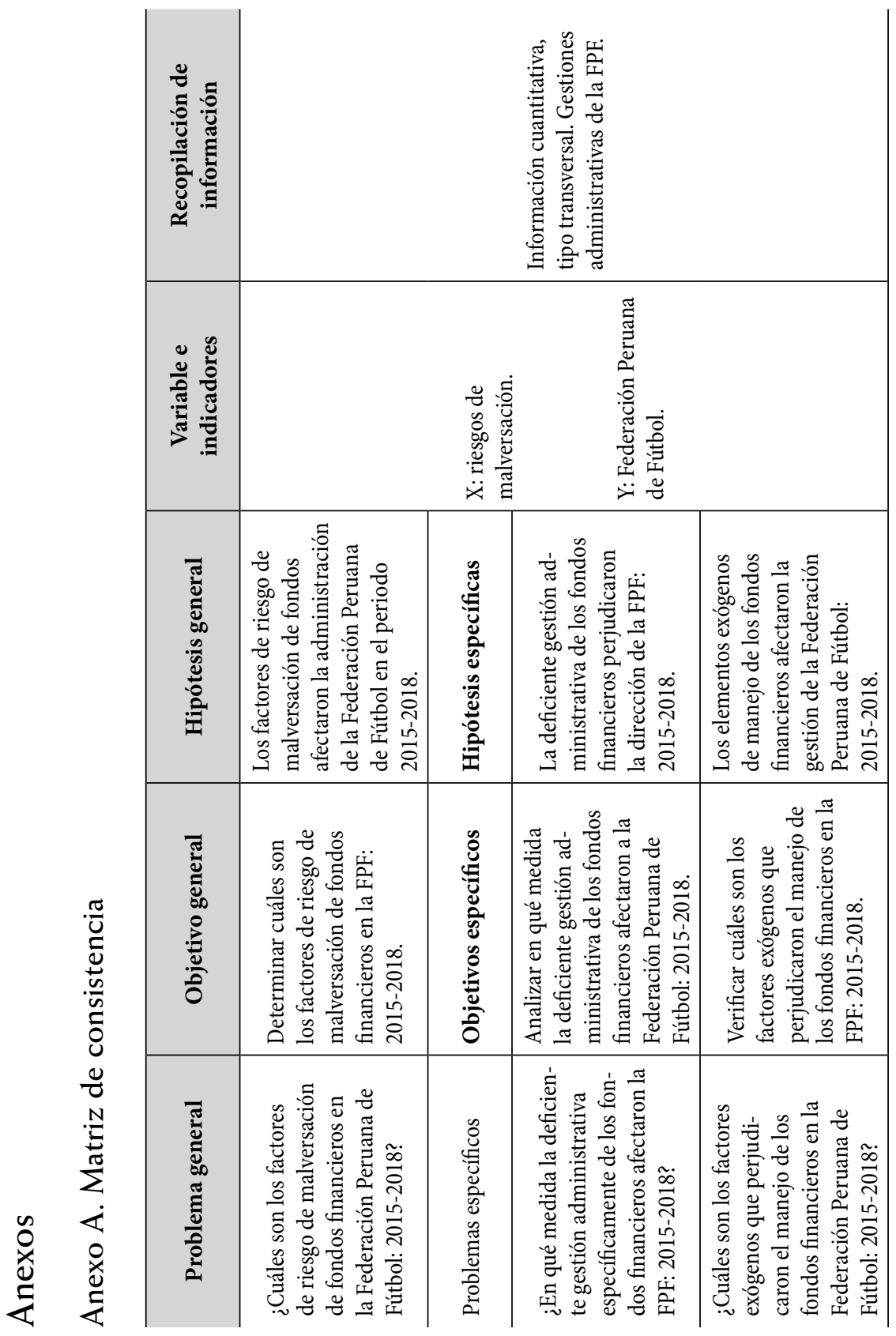




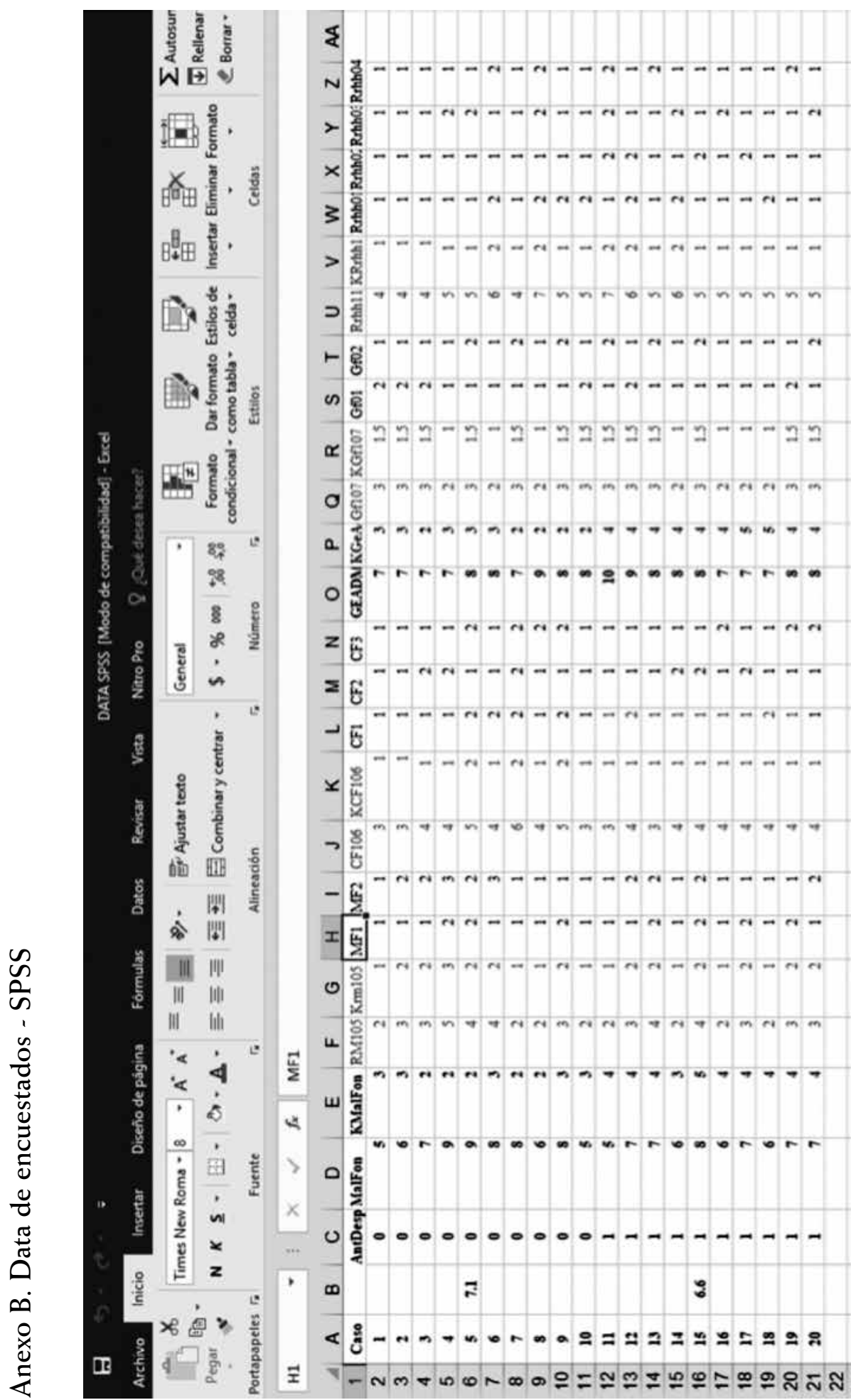




\section{Anexo C. Encuesta para procesar datos}

Sus comentarios son muy apreciados, muchas gracias por dedicar tiempo a participar en el cuestionario.

Indicaciones: marque con una equis $(\mathrm{X})$ la respuesta que mejor lo describa:

- Totalmente de acuerdo (T. A.).

- De acuerdo (D. A.).

- Ni de acuerdo, ni en desacuerdo, no lo sé (N. A. D.).

- En desacuerdo (E. D.).

- Totalmente en desacuerdo (T. D.).

\begin{tabular}{l|c|c|c|c|c}
\hline \multicolumn{1}{c|}{ Preguntas } & \multicolumn{4}{|c}{ Marque una alternativa } \\
\hline Sección 1 & $\begin{array}{c}\text { Totalmente } \\
\text { de acuerdo } \\
\text { (T. A.). }\end{array}$ & $\begin{array}{c}\text { De } \\
\text { acuerdo } \\
\text { (D. A). }\end{array}$ & $\begin{array}{c}\text { Ni de } \\
\text { acuerdo, } \\
\text { ni en } \\
\text { desacuerdo } \\
\text { (N. A. D.). }\end{array}$ & $\begin{array}{c}\text { En } \\
\text { desacuerdo } \\
\text { (E. D.). }\end{array}$ & $\begin{array}{c}\text { Totalmente } \\
\text { en } \\
\text { desacuerdo } \\
\text { (T. D.). }\end{array}$ \\
\hline $\begin{array}{l}\text { 1. ¿La alta dirección de } \\
\text { la FPF demuestra altos } \\
\text { estándares éticos? }\end{array}$ & & & & & \\
\hline $\begin{array}{l}\text { 2. ¿La alta dirección de } \\
\text { la FPF cumple con las } \\
\text { leyes, las normas y los } \\
\text { reglamentos que afectan } \\
\text { a la organización? }\end{array}$ & & & & & \\
\hline $\begin{array}{l}\text { 3. ¿Cree usted que los } \\
\text { directivos y empleados } \\
\text { son sensibles a las con- } \\
\text { sideraciones éticas y al } \\
\text { impacto y la percepción } \\
\text { de los demás a la hora } \\
\text { de tomar decisiones o } \\
\text { emprender acciones? }\end{array}$ & & & & & \\
\hline
\end{tabular}




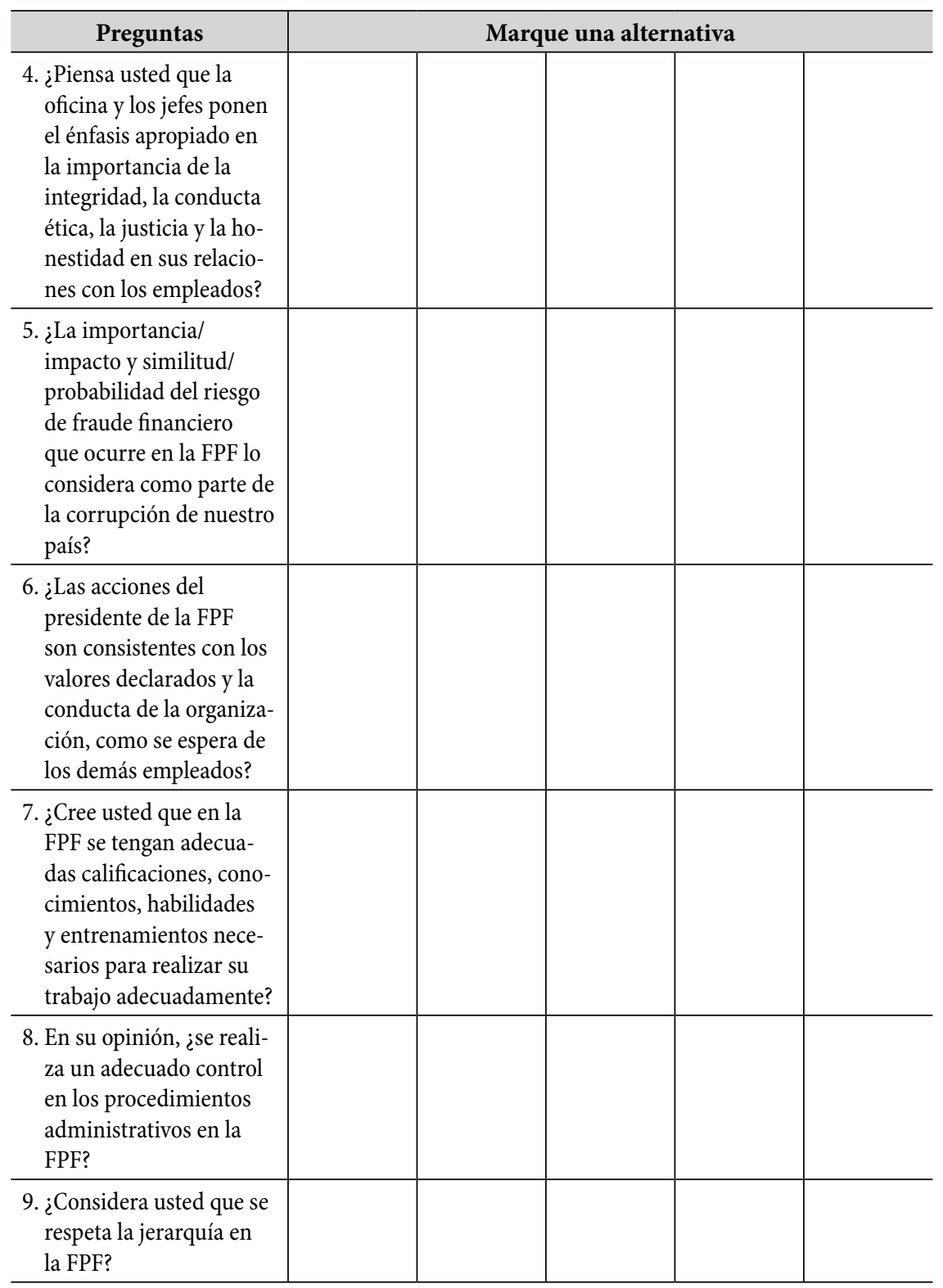


Factores de riesgo de malversación de fondos financieros

en la Federación Peruana de Fútbol: 2015-2018

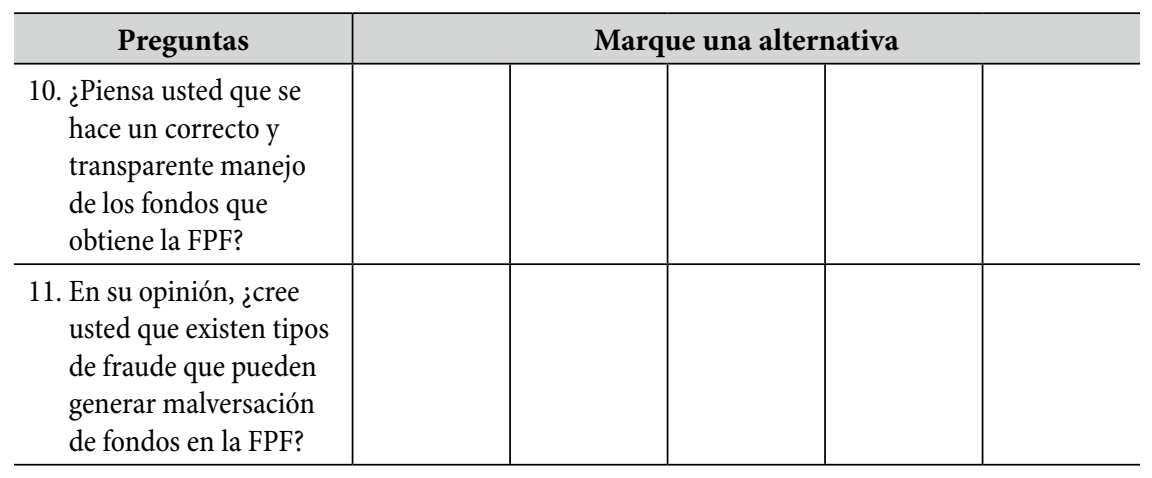

